\title{
EFEITO DO MÉTODO DE ABATE E DO SEXO SOBRE A QUALIDADE DA CARNE DE CAPIVARA (Hydrochaeris hydrochaeris) ${ }^{1}$
}

\author{
Sandra H. I. ODA², Maria C. BRESSAN ${ }^{2, *}$, Giulianna Z. MIGUEL ${ }^{2}$, Josye O. VIEIRA, \\ Peter B. FARIA3 ${ }^{3}$ Taciana V. SAVIAN ${ }^{4}$, Deyse M. KABEYA
}

\begin{abstract}
RESUMO
O presente trabalho teve como objetivo avaliar o efeito de dois métodos de abate (MA) e do sexo sobre a qualidade de carne de capivaras. Os métodos de abate avaliados foram o humanitário (MH) e por tiro (MT), sendo utilizados 2 lotes de 10 animais, um deles contendo

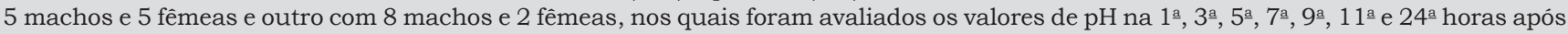
o abate e a perda de peso por cozimento (PPC), a cor e a força de cisalhamento (FC), em porções do músculo longissimus dorsi, retiradas entre a $10^{\mathrm{a}}$ e $12^{\mathrm{a}}$ vértebras. Os resultados obtidos indicaram que as médias de $\mathrm{pH}$ não foram influenciadas pelo MA e pelo sexo. Os valores médios foram: 6,$24 ; 6,05 ; 6,01 ; 6,01 ; 6,03 ; 6,04$ e 5,96 às $1,3,5,7,9,11$ e 24 horas, respectivamente. Em relação à cor, o MT apresentou valor de $L^{*}(32,40)$ superior $(\mathrm{P}<0,05)$ ao $\mathrm{MH}(29,59)$, não sendo observado o efeito de sexo sobre esse parâmetro. Nos demais parâmetros de cor e na PPC não foram observadas diferenças entre os lotes. A FC foi influenciada pelo MA, sendo observado maiores valores $(\mathrm{P}<0,05)$ para MT do que para $\mathrm{MH}$ com médias de 5,04 e 3,97kgf, respectivamente.
\end{abstract}

Palavras-chave: método de abate; sexo; carne; qualidade; pH; capivara.

\section{SUMMARY}

EFFECT OF SLAUGHTER METHOD AND GENDER ON THE MEAT QUALITY OF CAPYBARA (Hydrochaeris hydrochaeris L. 1766). The aim of this study was to assess the effect of two slaughter methods (SM) and the gender on the quality of capybara meat. The slaughter methods assessed were the humanitarian (HM) and by head-shot (HS). Two lots of 10 animals were used, one of them containing 5 males and 5 females and the other containing 8 males and 2 females. Values of pH of the longissimus dorsi muscle (between the vertebras $10^{\text {th }}$ and $12^{\text {th }}$ ) after $1,3,5,7,9,11$ and $24 \mathrm{~h}$ post mortem (p. m.), color (L, a e b), weight losses by cooking (WLC) and shear force $(\mathrm{SF})$ were assessed on samples obtained at these points. The results indicated that $\mathrm{pH}$ means were not influenced by both SM and sex. The medium values were: $6.24 ; 6.05 ; 6.01 ; 6.01 ; 6.03 ; 6.04$ and 5.96 at $1,3,5,7,9,11$ and 24 hours respectively. That demonstrated that in those encourage the installation of the rigor mortis superficial with reduced extension glicolitic. Regarding the color, HS presented higher $L^{*}(32.40)$ values $(P<0,05)$ than HM $(29.59)$, and the effect of gender on this parameter was not observed. On the other color parameters and on WLC no differences among the lots were observed. SF was influenced by SM, with higher values found $(\mathrm{P}<0,05)$ for the HS than for the HM, 5.04 and $3.97 \mathrm{kgf}$, respectively.

Keywords: slaughter method; sex; meat; quality; pH; capybara.

\section{1 - INTRODUÇÃO}

A necessidade de novas fontes protéicas para a população humana é de grande interesse social, pois atualmente as fontes de proteína animal disponiveis encontram-se concentradas na exploração de bovinos, suínos e aves. Nesse contexto, a utilização racional da fauna silvestre é um processo benéfico, que pode resultar em vantagens econômicas, sociais, e ao mesmo tempo proteger as espécies silvestres da extinção. Os fatores responsáveis pela redução da fauna são: a) a caça de subsistência, que apesar de sua grande importância na sobrevivência de populações ribeirinhas e tribos indígenas, tende a conduzir ao extermínio de espécies; b) a caça comercial, onde a matança de animais acontece de acordo com a exigência do mercado (carne, pele ou couro, plumas, ou ainda, animais vivos); e, c) a destruição dos habitats para dar lugar a exploração agropecuária.

\footnotetext{
1. Recebido para publicação em 28/06/2002. Aceito para publicação em 19/05/2004 (000917).

2. Departamento de Ciência dos Alimentos, Universidade Federal de Lavras/UFLA. Caixa Postal 37, CEP: 37200-000, Lavras, MG.

3. Departamento de Medicina Veterinária, Universidade Federal de Lavras/UFLA.

4. Departamento de Zootecnia, Universidade Federal de Lavras/UFLA.

5. Programa de Pós-graduação-Unicamp.

* A quem a correspondência deve ser enviada.
}

Entre as propostas de utilização da fauna silvestre de forma racional estão: a criação dos animais em cativeiro, a implantação da caça esportiva em fazendas com criatórios de animais silvestres, a implementação de um plano de manejo extensivo, o turismo ecológico, a exploração integrada de animais silvestres com o gado e o aproveitamento de terrenos marginais. Os animais silvestres podem se transformar em fontes renováveis de produtos de grande rentabilidade, contribuindo para a produção de alimentos e concorrendo, em custo de produção, com os animais domésticos. Dessa forma, a capivara surge como uma espécie de mamífero sul-americano com grande potencial zootécnico para a produção de carne e couro.

Atualmente, o mercado consumidor de carne tem se mostrado bastante receptivo ao consumo de carne de animais silvestres e exóticos. Apesar da existência de uma demanda pela carne de capivara nos grandes centros, e da possibilidade de abertura de novos mercados (mercado externo), os estudos de suas propriedades físico-químicas são escassos.

As características físico-químicas da carne, tais como: brilho, coloração, maciez, suculência e aroma, são parâmetros importantes para o consumidor no momento da compra e determinantes da aceitação global do corte e do tipo de carne, além de determinar a freqüência com que o consumidor vai adquirir esse produto. As características de qualidade de carne apresentam va- 
riações que estão relacionadas a vários fatores: método de abate [7, 8, 16]; espécie [9, 11]; idade de abate [17]; peso de abate [26, 27]; sexo [3]; manejo pré-abate [4, 18, 19]; e, manejo post mortem [4, 29]. Esses fatores influenciam a extensão e a velocidade da glicólise, bem como o valor de $\mathrm{pH}$ final. Em situações anormais, o $\mathrm{pH}$ final de carnes vermelhas pode ser igual ou mais elevado do que 6,2 (escura, firme e ressecada na superficie) e essa carne tem vida-de-prateleira curta. Por outro lado, também em situações anormais, o pH da carne pode apresentar um rápido declínio (valores iguais ou inferiores a 5,8, 1 hora p.m.), que associado à temperatura de carcaça elevada $\left(36^{\circ} \mathrm{C}\right)$ causa desnaturação protéica, baixa capacidade de retenção de água, coloração pálida e baixa aptidão para a transformação [6].

O objetivo do presente trabalho foi: acompanhar a taxa de acidificação das carnes nas primeiras 24 horas post mortem, através da medida de $\mathrm{pH}$; avaliar a cor; a perda de peso por cozimento (PPC) e a maciez (força de cisalhamento) em carnes de capivaras oriundas de duas modalidades de abate (humanitário e por tiro) de diferentes sexos.

\section{2 - MATERIAIS E MÉTODOS}

\section{1 - Material}

Foram utilizadas 20 capivaras $(13$ machos e 7 fêmeas) com idade de 1 ano e peso vivo médio de 45,71 kg, provenientes de um mesmo zoocriadouro, cuja criação foi em Sistema Intensivo de Criação ao Ar Livre (SISCAL). Foram abatidas em julho de 2001, no frigorífico da Empresa Pró-Fauna Assessoria e Comércio Ltda. (IBAMA, no 1-35-93-0848-0), com sede em Iguape (SP).

\section{2 - Tratamentos}

No desembarque, as capivaras foram separadas aleatoriamente em 2 lotes de 10 animais, sendo o primeiro composto por 5 fêmeas e 5 machos e o segundo por 2 fêmeas e 8 machos. Os animais foram submetidos a jejum e dieta hídrica de 48 h e tempo de descanso de $24 \mathrm{~h}$ em baias coletivas. O primeiro lote foi submetido ao abate humanitário (MH) (Decreto 2244 de 04/06/ 1997), em que os animais foram insensibilizados (300 V, 2 A por 5s) e sangrados após 15 segundos. O segundo lote de animais foi submetido ao método de abate por tiro na região temporo-occipital (MT) e após 10min, foram conduzidos ao abatedouro e sangrados. Esse método corresponde ao que é indicado pela legislação de alguns países que permitem a caça esportiva, sendo portanto, diferente dos métodos de abate convencionais preconizados pela legislação brasileira. A partir dessas operações, os animais seguiram na linha de abate, onde foram realizados: escaldagem $\left(60^{\circ} \mathrm{C}\right)$, pelagem, evisceração, inspeção (SIF), serragem longitudinal da carcaça e resfriamento a $4 \pm 1^{\circ} \mathrm{C}$.

\section{3 - Análises laboratoriais}

As leituras de $\mathrm{pH}$ foram realizadas no músculo longissimus dorsi (LD) às $1,3,5,7,9,11$ e 24h post mortem (p.m.), no mesmo animal, com auxílio de um potenciômetro portátil (Digimed M DM20) equipado com eletrodo de inserção, com resolução de 0,01 unidades de $\mathrm{pH}$. Foram obtidas 3 leituras para cada horário, sendo utilizada, na análise estatística, o valor médio desses resultados.

As amostras para as determinações da cor, perda de peso por cozimento (PPC) e força de cisalhamento (FC) foram coletadas da porção torácica do músculo LD (porção cranial do corte denominado "carré", entre a 6a e $12^{a}$ vértebras) das meias-carcaças esquerda e direita. Depois de embaladas em sacos plásticos, as amostras foram identificadas e congeladas a $-12^{\circ} \mathrm{C}$ e, no momento das análises, foram descongeladas a $3,5 \pm 0,5^{\circ} \mathrm{C}$.

A cor dos cortes foi avaliada pelo sistema CIE L*a* $b^{*}$ $\left(L^{*}=\right.$ luminosidade; $a^{*}=$ teor de vermelho; $e, b^{*}=$ teor de amarelo). A medida de cor foi realizada com colorímetro (Minolta Chroma Meter, M CR-300b), calibrado para um padrão branco em ladrilho [4]. As amostras foram seccionadas, expondo-se a superfície do corte ao ar por $30 \mathrm{~min}$, antes da leitura. As leituras foram realizadas em 3 fatias, cortadas no sentido transversal do músculo LD, sendo que em cada fatia foram analisados 3 pontos distintos. O valor médio foi utilizado na análise estatística.

A PPC foi determinada conforme descrição de AMSA [1]. As amostras foram identificadas, pesadas em balança semi-analitica (Hobart-Dayton M 14239), embaladas em papel alumínio e cozidas em chapa a $150^{\circ} \mathrm{C}$ até atingir a temperatura interna de $72 \pm 2{ }^{\circ} \mathrm{C}$ (monitorada com termômetro digital). Após o cozimento, as amostras foram resfriadas à temperatura ambiente e novamente pesadas. A diferença entre peso inicial e final correspondeu a PPC. As análises foram realizadas em triplicata.

Após a determinação da PPC, as amostras cozidas foram utilizadas para a análise de FC. Foram retirados 3 cilindros de carne por fatia, de aproximadamente $1,5 \mathrm{~cm}$ de comprimento, $1,0 \mathrm{~cm}$ de largura e $0,2 \mathrm{~cm}$ de espessura, de acordo com WHEELER \& KOOHMARAIE [28], com auxílio de uma faca afiada, totalizando 9 cilindros por amostra. Os cilindros, livres de gorduras e nervos, foram retirados no sentido da fibra. A FC foi registrada em texturômetro, acoplado a uma "probe" WarnerBratzler numa escala variando de 0 a $10 \mathrm{kgf} / \mathrm{s}$.

\section{4 - Delineamento experimental e análise estatís- tica}

O delineamento experimental foi inteiramente casualizado em esquema fatorial $2 \times 2$, sendo dois métodos de abate (MA): humanitário (MH) e por tiro (MT); e, dois sexos: macho e fêmea. Os resultados foram analisados no programa estatístico SAS versão 6.12 [22]. Quando a análise de variância identificou diferenças, os dados foram submetidos ao teste de T. O modelo experimental para as análises de cor, PPC e FC foi:

$$
\text { Yijk }=\mu+M i+S j+(M S) i j+P+\text { Eijk. }
$$

Onde: 
Yijk = observação no músculo longissimus dorsi de capivaras, abatidas pelo método $i$, do sexo $j$, na repetição $\mathrm{k}$;

$\mu=$ média geral do experimento;

Mi = efeito do método de abate $\mathrm{i}$, sendo $\mathrm{i}=1,2$;

$\mathrm{Sj}=$ efeito do sexo $\mathrm{j}$, sendo $\mathrm{j}=1,2$;

$(\mathrm{MS}) \mathrm{ij}$ = efeito da interação do método de abate i com o sexo $\mathrm{j}$;

$\mathrm{P}=$ covariável de peso ao abate;

Eijk = erro experimental associado à observação Yijk, normalmente distribuída, com média 0 e variância $\sigma^{2}$

A análise estatística de $\mathrm{pH}$ foi realizado em delineamento inteiramente casualizado em esquema fatorial 2x2, sendo dois métodos de abate (MA): humanitário $(\mathrm{MH})$ e por tiro (MT); e, dois sexos: macho e fêmea sendo as parcelas subdivididas no tempo (hora da medida). Após, os dados foram submetidos à análise de regressão pelo programa estatístico Table Curve v. 2.03 (Jandel Scientific, incorporation) e Fcalc 32 for Windows V.11. O modelo experimental para as medidas de $\mathrm{pH}$ foi:

Yijkl $=\mu+\mathrm{Mi}+\mathrm{Sj}+(\mathrm{MS}) \mathrm{ij}+\mathrm{Tk}+(\mathrm{MT}) \mathrm{ik}+(\mathrm{ST}) \mathrm{jk}+$ (MST)ijk + Eijkl

Onde:

Yijkl = valor de $\mathrm{pH}$ do método de abate $\mathrm{i}$ no sexo $\mathrm{j}$, no horário de medição $\mathrm{k}$, no sexo $\mathrm{k}$, na repetição 1 ;

$\mu=$ constante associada a todas as observações;

$\mathrm{Mi}=$ efeito do método de abate, sendo $\mathrm{i}=1,2$;

$\mathrm{Sj}=$ efeito do sexo $\mathrm{j}$, sendo $\mathrm{j}=1,2$;

$(\mathrm{MS}) \mathrm{ij}$ = efeito da interação do método de abate i com o sexo j;

$\mathrm{Tk}=$ efeito do horário de medição $\mathrm{k}$, sendo $\mathrm{k}=1,2,3$, 4, 5, 6, 7 (para valores de $\mathrm{pH}$ );

(MT)ik = efeito da interação entre o método de abate $\mathrm{i}$ e o horário de medição do $\mathrm{pH}$ k;

(ST)jk = efeito da interação entre sexo j e o horário de medição do $\mathrm{pH} \mathrm{k}$;

$(\mathrm{MST}) \mathrm{ijk}=$ efeito da interação entre metodo de abate $\mathrm{i}$, do sexo j e horário de medição do $\mathrm{pH} \mathrm{k}$;

Eijkl = erro associado à observação Yijkl, normalmente distribuída, com média 0 e variância $\sigma^{2}$.

\section{3 - RESULTADOS E DISCUSSÃO}

\section{1 - Declinio do pH post mortem (p.m.)}

A curva ajustada de pH (Figura 1) mostrou comportamento exponencial e coeficiente de determinação de $\mathrm{R}^{2}=91,93$, com uma queda de $\mathrm{pH}$ mais acentuada nas primeiras 5 horas p.m., seguida da tendência de estabilização e efeito significativo $(\mathrm{P}<0,01)$ para os horários de leitura.

A análise de variância não mostrou efeito do MA e do sexo sobre os valores de $\mathrm{pH}$. Com relação ao fator sexo, esses dados concordam com os resultados relatados por JARDIM [9] que, trabalhando com capivaras de diferentes faixas de peso ao abate (40-50kg e 50-60kg), observou valores semelhantes de $\mathrm{pH}$ entre machos e fêmeas nos diferentes horários (2, 5, 8 e 24 horas) após o abate. Em ovinos, SOUZA [26], analisando a evolução do pH p.m. em animais machos e fêmeas, abatidos com 15, 25, 35 e 45kg de peso vivo, também não encontrou diferença para sexo, confirmando os resultados citados por VERGARA, MOLINA \& GALLEGO [27] para esta espécie.

Com o objetivo de determinar os efeitos do manejo pré-abate sobre alguns parâmetros de qualidade da carne de cervos, POLLARD et al. [16] compararam animais abatidos a tiro com animais submetidos ao abate comercial e nas três medidas de $\mathrm{pH}$ realizadas após o abate (semanas 0, 3 e 5) e observaram que o pH encontrado em músculos de animais abatidos por tiro mostrou-se mais elevado do que no método humanitário. Contrariando os resultados de POLLARD et al. [16], no presente trabalho, os métodos de abate não afetaram o declínio de $\mathrm{pH}$ e o $\mathrm{pH}$ final obtido no músculo LD.

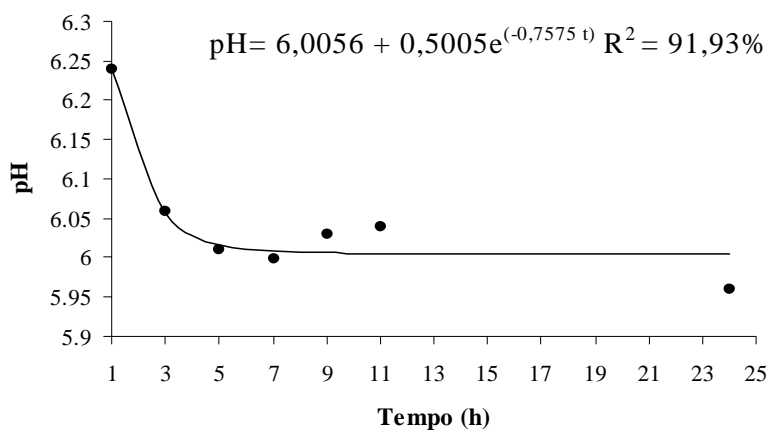

FIGURA 1. Valores de pH do músculo LD de capivaras, nas primeiras 24 horas após o abate.

Com relação ao declínio de $\mathrm{pH}$ em capivaras, JARDIM [9] relatou valores elevados ( $>6,2$ às 8 h e de 5,94 a 6,04 às 24 h p.m.). Esse autor descreveu que no préabate os animais viajaram por distância longa e que, uma vez nas baias de descanso (com capacidade para 3 capivaras), os mesmos apresentaram-se agitados e, em alguns casos, foram observadas brigas. Outro trabalho subseqüente foi realizado em capivaras por MIGUEL [10] e na tentativa de anular os efeitos de estresse pré-abate, os animais foram abatidos nas próprias baias onde eram mantidos (sem transporte e sem jejum), por tiro na região temporo-ocipital. Nesse caso, o pH médio de 5,89 foi observado às $4 \mathrm{~h}$, e de 5,74 às $24 \mathrm{~h}$ p.m. (normalmente, o pH de 5,9 é utilizado como indicativo da instalação do rigor [6]). Com isso, fica explícito que, no trabalho de JARDIM [9], os animais foram abatidos em condição de estresse e que os animais dessa espécie, quando não submetidos a condições adversas [10] mostram declínio de $\mathrm{pH}$ semelhante ao observado em espécies como: suínos (5,6-5,7 às 6-8h p.m. [20]); e, ovinos (5,8 às 8 h p.m. [17]). No presente trabalho, o pH final médio foi de 5,96 (um valor que é considerado acima do intervalo adequado de acidificação em carnes vermelhas, que é de 5,4 a 5,8 [6,18]), revelando que, 
certamente, houve um gasto das reservas de glicogênio muscular no pré-abate, desencadeando carnes menos ácidas às 24 horas post mortem.

\section{2 - $\operatorname{Cor}\left(L^{*}, a^{*} \mathbf{e} b^{*}\right)$}

A análise de variância não identificou efeitos significativos dos fatores sobre os valores médios dos indices de cor $\mathrm{a}^{*} \mathrm{e} \mathrm{b}^{*}$ (Tabela 1). Isso demonstrou que o valor $\mathrm{a}^{*}$ (teor de vermelho) e o valor $\mathrm{b}^{*}$ (teor de amarelo) foram semelhantes entre capivaras $\mathrm{M}$ e $\mathrm{F}$ e entre capivaras abatidas pelos métodos $\mathrm{MH}$ e MT. O fator sexo não influenciou os resultados de $\mathrm{L}^{*}$ (luminosidade). Entretanto, houve diferença $(\mathrm{P}<0,05)$ entre os valores do índice de $\mathrm{L}^{*}$ entre capivaras abatidas pelo MH e MT. A interação entre os fatores sobre os componentes de cor não foi significativa.

No método $\mathrm{MH}$ foram observadas amostras mais escuras $(29,58)$ do que aquelas verificadas no MT $(32,40)$. Em capivaras submetidas ao abate convencional (animais criados no sistema SISCAL, submetidos a descanso, atordoamento, seguido da sangria) JARDIM [9] encontrou valor médio de luminosidade de 34,28 e dado semelhante $(34,76)$ foi relatado por MIGUEL [10] em capivaras (mantidas em Sistema Intensivo de Criação em Confinamento) submetidas ao abate por tiro, seguido de sangria. Considerando os animais do presente trabalho (oriundos do mesmo zoocriatório, semelhante sistema de criação, faixa de peso de abate comercial, tempo de descanso semelhante), o esperado seria que as médias de luminosidade fossem semelhantes ou no MT, a carne se apresentasse mais escura, do que no $\mathrm{MH}$ devido à demora de 15 minutos para a realização da sangria. Entretanto isso não foi encontrado.

Normalmente, a luminosidade está associada com: a) a quantidade de água no tecido; SOUZA [26] e BONAGURIO [3] descreveram que animais de $15 \mathrm{~kg}$ (com maior umidade na carne) mostraram maior luminosidade do que animais mais velhos de 25, 35 e 45kg (com menor percentual de água e maior percentual de extrato etéreo); b) a evolução das reações bioquímicas post mortem; BRESSAN [4], analisando as correções entre valor $\mathrm{R}$ (relação entre as absorbâncias de extrato de músculo a 250nm e 260nm, que avalia a quantidade de inosina para trifosfato de adenosina, cuja relação descreve a instalação do rigor) e a luminosidade, e a correlação entre $\mathrm{pH}$ e luminosidade, relatou que o valor $\mathrm{L}^{*} \mathrm{e}$ positivamente correlacionado com o valor $R(r=0,53$, $\mathrm{P}=0,01$ ou seja, quanto maior a hidrólise de ATP, maior a luminosidade) e inversamente correlacionado com o $\mathrm{pH}(\mathrm{r}=-0,64, \mathrm{P}=0,01 \%$ ou seja, quanto menor o valor de $\mathrm{pH}$ maior a luminosidade). A premissa de que o $\mathrm{pH}$ está relacionado com a capacidade de retenção de água no músculo $[6,18,29]$ e, por conseqüência na medida que descreve essa luminosidade ou brilho, no caso desse experimento, não esclarece o comportamento dos valores médios de L*obtidos nos tratamentos MH e MT.

$\mathrm{O} \mathrm{L}^{*}$ apresenta variações quando comparado as diferentes espécies: em avestruz, PALEARI et al. [12] relatam médias de 36,73; em bovinos adultos, PICALLO et al. [15] citam médias entre 23,98-25,64; em bovinos jovens (novilhos), PEREIRA et al. [14] citam L* de 41,842,3; em ovinos, SOUZA [26] reporta valores de 32,8935,25; e, BONAGURIO [3], médias de 35,30-38,88; e em suínos, SILVEIRA [25] relata médias de 49,05-50,21. Comparando os resultados do presente trabalho com os valores dos diferentes autores, observa-se que a luminosidade em capivaras foi inferior a observada em suínos e aves (carnes de coloração clara, com L* de 43,04 a 50,21). Essa comparação mostra que o músculo LD de capivaras apresenta valores de luminosidade que se aproxima aos verificados para carnes vermelhas (com variação de $28,78-41,8)$.

TABELA 1. Médias e erros padrão (EP) dos teores de L*a*b*, e valores de PPC e FC encontrados no músculo LD de capivaras.

\begin{tabular}{ccccc}
\hline & \multicolumn{2}{c}{ Sexo } & \multicolumn{2}{c}{ Método de Abate } \\
\cline { 2 - 5 } & Macho & Fêmea & Humanitário & Tiro \\
\hline $\mathrm{L}^{\star}$ & $31,90^{\mathrm{a}} \pm 0,73$ & $30,10^{\mathrm{a}} \pm 1,00$ & $29,59^{\mathrm{b}} \pm 0,90$ & $32,40^{\mathrm{a}} \pm 0,86$ \\
$\mathrm{a}^{\star}$ & $14,10^{\mathrm{a}} \pm 0,75$ & $14,33^{\mathrm{a}} \pm 1,05$ & $14,72^{\mathrm{a}} \pm 0,94$ & $13,69^{\mathrm{a}} \pm 0,88$ \\
$\mathrm{~b}^{\star}$ & $0,34^{\mathrm{a}} \pm 0,27$ & $0,69^{\mathrm{a}} \pm 0,37$ & $0,36^{\mathrm{a}} \pm 0,34$ & $0,67^{\mathrm{a}} \pm 0,32$ \\
PPC (\%) & $29,06^{\mathrm{a}} \pm 2,11$ & $28,21^{\mathrm{a}} \pm 3,32$ & $24,93^{\mathrm{a}} \pm 3,04$ & $32,33^{\mathrm{a}} \pm 2,46$ \\
FC (kgf) & $4,43^{\mathrm{a}} \pm 0,27$ & $4,59^{\mathrm{a}} \pm 0,36$ & $3,97^{\mathrm{b}} \pm 0,33$ & $5,05^{\mathrm{a}} \pm 0,31$ \\
\hline
\end{tabular}

Médias seguidas da mesma letra são estatisticamente iguais entre si pelo teste de $\mathrm{T}$, ao nivel de $5 \%$ de probabilidade $(\mathrm{P}<0,05)$.

Os teores de vermelho (a*) variaram de 13,43 a 14,74. Em lombos de capivaras, são reportados valores de 9,6412,77 [9] e de 14,39-19,25 [10]. Em animais silvestres, são citados valores de 16 a 20 para cervos [16]; 11,42 para impalas [7]; e, 22,84 em avestruzes [12]. Em espécies domésticas, foram relatados teores de 5,50-5,94 em suínos [25]; 10,0 a 18,01 em ovinos [17, 26]; 15,5 em bovinos [14]; e de 1,9 a 3,0 em peitos de frangos [5]. Comparando-se os resultados obtidos com os diferentes autores, é possível estabelecer que a carne de capivara do presente trabalho assemelhou-se às carnes vermelhas, cujos teores de vermelho foram superiores em relação às carnes brancas.

Os teores de amarelo (b*) variaram de 0,65 a 0,73. Em capivaras, são reportadas variações de 1,31 a 2,50 [9] e de 0,04 a 2,07 [10]. Em espécies de caça, foram citadas médias de 6,57 em avestruzes [12]; e de 7,0-7,62 em impalas [7]. Nas espécies domésticas, foram reportadas médias mais elevadas por CONTRERAS [5]; SILVEIRA [25]; e, PEREIRA et al. [14] para peitos de frangos $(4,1-5,6)$; coxas de frangos $(6,14)$; suínos $(5,80-6,53)$; e, bovinos $(13,8)$. Em geral, o b* avalia os pigmentos carotenóides depositados na gordura da carne. Possivelmente, os reduzidos valores de $b^{*}$ sejam conseqüência do baixo teor de gordura que caracteriza as carnes de animais silvestres [23].

Comparando-se os dados do presente trabalho aos encontrados na literatura para outras espécies, observase que a carne de capivara apresentou índices de luminosidade baixos e elevados teores de vermelho, assemelhando-se a carnes de bovinos e ovinos (carnes vermelhas). 


\section{3 - Perda de peso por cozimento (PPC) e força de cisalhamento (FC)}

A análise de variância não identificou efeito dos MA e do sexo sobre os valores de PPC e FC (Tabela 1). Entretanto, o MA influenciou $(\mathrm{P}<0,05)$ a $\mathrm{FC}$, de forma que no $\mathrm{MH}$ foi observada menor $\mathrm{FC}(3,97 \mathrm{kgf})$ do que no MT (5,04kgf). A interação entre os fatores sobre a PPC e FC não foi significativa.

Os valores de PPC variaram de 24,93 a 33,84\%. Em LD de capivaras são reportados valores de 29,87\% [10] e 32,27\% [9]. Em outras espécies de animais silvestres e exóticos, são relatados valores de 23,48-24,48\% em impalas [7]; de 21,9\% em zebras; e, de $36,4 \%$ em oryx (Oryx beisa) [11]. Em espécies domésticas, foram descritas médias de 38,23-40,48\% em ovinos abatidos aos 15, 25, 35 e 45kg [17]; de 27,17-36,63\% em suínos [25]; e de $27,2 \%$ em aves [4]. A comparação entre os dados de capivaras e as variações descritas na literatura revela que a PPC em capivaras mostrou valores próximos aos valores relatados para ovinos e suínos. FORREST et al. [6] e PARDI et al. [13] descreveram que a PPC, em animais de açougue pode variar de 20 a $40 \%$ e os fatores que interferem nos resultados são: metodologias de cocção; preparo da amostra e categorias de pesos ao abate.

As carnes de animais abatidos pelo $\mathrm{MH}$ (3,97kgf) foram mais macias do que no MT (5,04kgf). Esses resultados contrariam a afirmação de que a velocidade de instalação do rigor influencia na maciez de forma que, quanto maior o pH final, maior a dureza na carne [19]. Por outro lado, esses resultados confirmam as observações de SILVA, PATARATA \& MARTINS [24], que observaram valores de $\mathrm{FC}$ superiores nas carnes de $\mathrm{pH}$ normal $(\mathrm{pH}=5,7)$ em relação às $\mathrm{FC}$ em carnes DFD moderado $(\mathrm{pH}=6,1)$ e DFD $(\mathrm{pH}>6,5)$, e atribuíram esse resultado à maior atividade das calpaínas em valores de $\mathrm{pH}$ próximos à neutralidade.

Em capivaras, são relatados valores de FC de 4,945,50kgf [9]; de 5,16kgf [10]; e, de 4,30-4,70kgf [21]. Entretanto, valores mais elevados foram relatados em cervos (8-12kgf) [16] e em ovinos Santa Inês (6,07-17,17kgf) [3]. A carne é classificada como macia quando a FC atinge valores de até 8kgf; é considerada aceitável quando esses valores estão entre $8 \mathrm{kgf}$ e $11 \mathrm{kgf}$; e, é considerada dura com valores acima de $11 \mathrm{kgf}$ [2]. Considerando esses limites e os valores de FC, a carne de capivara, neste experimento, pode ser classificada como macia.

Considerando os parâmetros de qualidade estudados em relação à proposta de abate por tiro (uma modalidade esportiva, que retarda o tempo entre o tiro e a sangria e poderia prejudicar a eficiência dessa sangria [4] e a cor da carne, pois o sangue contém pigmentos hemínico, a hemoglobina, que, segundo WARRIS [29], contribui de modo importante na concentração total dos pigmentos de cor), observa-se que os componentes da cor: teor de vermelho e teor de amarelo não são afetados e a luminosidade (que, nesse caso, poderia apresentar-se mais escura, com um valor médio de $L^{*}$ mais baixo) mostrou-se mais elevada, ou seja, carne mais clara sem, contudo, atingir valores que determinariam carnes anômalas. As demais características como $\mathrm{pH}$ e PPC não demonstram diferenças entre o abate convencional e o abate por tiro. A maciez foi maior no método humanitário (menor FC), porém, a FC das carnes oriundas de animais abatidos por tiro mostrou-se dentro de uma faixa de FC considerada como macia.

\section{4 - CONCLUSÕES}

Com base nos resultados encontrados no presente trabalho, é possivel inferir que: a) o método de abate por tiro ocasiona carnes mais claras do que no método convencional; entretanto, os outros componentes da cor não são alterados pelo método de abate; b) os métodos de abate e o sexo não influenciaram a perda de peso por cozimento; e, c) o método convencional de abate proporciona carnes mais macias, do que o método por tiro, porém, em ambos os métodos a carne foi macia, considerando os limites de FC adotados na literatura. Assim, o método de abate por tiro (uma modalidade esportiva), que operacionalmente provocaria um retardo de tempo no processo entre o tiro e a sangria, não provoca alterações significativas na qualidade de carne.

\section{5 - REFERÊNCIAS BIBLIOGRÁFICAS}

[1] AMSA. Guidelines for cooking and sensory evaluation of meat. Chicago: AMSA, 1978.

[2] BICKERSTAFFE, R.; LE COUTER, C.E.; MORTON, J.D. Consistency of tenderness in New Zealand retail meat. $43^{\text {rd }}$ Internacional Congress of Meat Science and Technology, Auckland, New Zealand, 27 July-1 August, 1997.

[3] BONAGURIO, S. Qualidade da carne de cordeiros Santa Inês puros e mestiços com Texel abatidos com diferentes pesos. Lavras, 2001. 150p. (Dissertação de Mestrado em Zootecnia) - Universidade Federal de Lavras (UFLA).

[4] BRESSAN, M.C. Efeitos dos fatores pré e pós-abate sobre a qualidade da carne de peito de frango. Campinas, 1998. 201p. (Tese de Doutorado) - Universidade Estadual de Campinas (UNICAMP).

[5] CONTRERAS, C.J.C. Efeitos do atordoamento elétrico, estimulação elétrica e da desossa à quente na qualidade da carne do peito de frango "pectoralis major". Campinas, 1995. 150p. (Tese de Doutorado) Universidade Estadual de Campinas (UNICAMP).

[6] FORREST, J.C.; ABERLE, E.D.; HEDRICK, H.B.; JEDGE, M.D.; MERKEL, R.A. Fundamentos de ciencia de la carne. Zaragoza, Acribia, 1979.

[7] HOFFMAN, L.C. Meat quality attributes of night-cropped Impala (Aepyceros melampus). South African Journal of Animal Science, v. 30, n. 2, p. 133-137, 2000.

[8] HOFFMAN, L.C.; FERREIRA, A.V. pH decline of the M. longissimus thoracis of night-cropped Grey Duiker (Sylvicapra grimmia). South African Journal Animal Science, v. 30, n. 1, p. 16-17, 2000.

[9] JARDIM, N.S. Sexo e diferentes pesos ao abate na qualidade da carne de capivara (Hydrochaeris hydrochaeris L. 1766). Lavras, 2001. 119p. (Dissertação de Mestrado) - Universidade Federal de Lavras (UFLA).

[10] MIGUEL, G.Z. Caracterização da carcaça e da carne de capivaras (Hydrochaeris hydrochaeris L. 1766) em 
idade adulta. Lavras, 2002. 107p. (Dissertação de Mestrado) - Universidade Federal de Lavras (UFLA).

[11] ONYANGO, C.A.; IZUMimoto, M.; KUTIMA, P.M. Comparison of some physical and chemical properties of selected game meats. Meat Science, v. 49, n. 1, p. 117-125, 1998.

[12] PAlEARI, M.A.; CAMISASCA, S.; BERETTA, G.; RENAN, P.; CORISCO, P.; BERTOLO, G.; CRIVELLI, G. Ostrich meat physico chemical characteristics and comparison with turkey and bovine meat. Meat Science, v. 48, n. 3-4, p. 205-210, 1998.

[13] PARDI, M.C.; SANTOS, I.F.; SOUZA, E.R.; PARDI, H.S. Ciência, higiene e tecnologia da carne: tecnologia da sua obtenção e transformação. Goiânia: Universidade de Goiás, 1993, v. 1. 623p.

[14] PEREIRA, A.S.C.; SOBRAL, P.J.A.; SILVA, S. L.; LEME, P.R. Características físico-químicas do contra-filé congelado de novilhos nelore (Bos indicus) suplementados com vitamina E. In: I Congresso Brasileiro de Ciência e Tecnologia de Carnes, São Pedro, SP, 22 a 25 de outubro de 2001.

[15] PICALLO, A.B.; SANCHO, A.M.; MARGARÍA, C.A.; LASTA, J.A. Colour and tenderness relationships in different steer breeds. In: $44^{\text {th }}$ International Congress of Meat Science and Technology, Barcelona/ Spanien, 30 August-4 September, 1998.

[16] POLLARD, J.C.; LITTLEJOHN, R.P.; ASHER, G.W.; PEARSE, A.J.T.; STEVENSON-BARRY, J.M.; McGREGOR, S.K.; MANLEY, T.R.; DUNCAN, S.J.; SUTTON, C.M.; POLLOCK, K.L.; PRESCOTT, J. A comparison of biochemical and meat quality variables in red deer (Cervus elaphus) following either slaughter at pasture or killing at a deer slaughter plant. Meat Science, v. 60, p. 85-94, 2002.

[17] PRADO, O.V. Qualidade de carne de cordeiros Santa Inês e Bergamácia abatidos em diferentes pesos. Lavras, 1999. 109p. (Dissertação de Mestrado) - Universidade Federal de Lavras (UFLA).

[18] PRÄNDAL, O.; FISCHER, A.; SCHMIDHOFER, T.; SINELL, H.J. Tecnologia e hygiene de la carne. Zaragoza: Acribia, 1994. 854p.

[19] PURCHAS, R.W.; YAN, X.; HARTLEY, D.G. The influence of a period of ageing on the relationship between ultimate $\mathrm{pH}$ and shear values of beef $M$. longissimus thoracis. Meat Science, v. 51, p. 135-141, 1999.

[20] ROÇA, R.O.; SERRANO, A.M. Abate de bovinos: conversão do músculo em carne. Revista Higiene Alimentar, n. 33, p. 7, 1994.

[21] SALDANHA, T. Determinação da composição centesimal nos diferentes cortes da carne de capivara (Hydrochoerus hydrochaeris). Rio de Janeiro, 2000. 105p. (Dissertação de Mestrado) - Universidade Federal Rural do Rio de Janeiro (UFRRJ).

[22] SAS INSTITUTE. SAS user's guide: statistics. 5ed. Cary, North Carolina, 1985. 956p.

[23] SINCLAIR, A. J.; O'DEA, K. Fats in Human diets through history: is the western diet out of step? In: WOOD, J. D.; FISHER, A. V. Reducing fat in meat animals. London: Elsevier, 1990, p. 1-47.

[24] SILVA, J.A.; PATARATA, L.; MARTINS, C. Influence of ultimate $\mathrm{pH}$ on bovine meat tenderness during ageing. Meat Science, v. 52, p. 453-459, 1999.

[25] SILVEIRA, E.T.F. Técnicas de abate e seus efeitos na qualidade da carne suina. Campinas, 1997. 226p. (Tese de Doutorado) - Universidade Estadual de Campinas (UNICAMP).

[26] SOUZA, X.R. Efeitos de grupo genético, sexo e peso ao abate na qualidade de carne de cordeiros em crescimento. Lavras, 2001. 116p. (Dissertação de Mestrado) - Universidade Federal de Lavras (UFLA).

[27] VERGARA, H.; MOLINA, A.; GALLEGO, L. Influence of sex and slaughter weight on carcass and meat quality in light and medium weight lambs produced in intensive systems. Meat Science, v. 52, p. 221-226, 1999.

[28 WHEELER, T.L.; KOOHMARAIE, M. Prerigor and postrigor changes in tenderness of ovine longissimus muscle. Journal of Animal Science, v. 72, p. 1232-1238, 1994

[29] WARRIS, P.D. Ciencia de la carne. Zaragoza: Acribia, 2003. 309p

\section{6-AGRADECIMENTOS}

Os autores agradecem: ao CNPq pela concessão de recursos para o desenvolvimento do projeto; a Universidade Federal de Lavras pela concessão de bolsa de trabalho a aluna Josye O. e Vieira; e, a Empresa Pró-Fauna Assessoria e Comércio Ltda. pela doação de amostras. 